\title{
VALIDATION OF A KNEE ANGLE MEASUREMENT SYSTEM BASED ON IMUS
}

\author{
NUNO FERRETE RIBEIRO ${ }^{1}$, CÉSAR FERREIRA ${ }^{1}$, LUIS PAULO REIS ${ }^{2}$, HÉLDER \\ SILVA $^{1}$, PEDRO MACEDO ${ }^{1}$, LUÍS ROCHA ${ }^{1}$ and CRISTINA P. SANTOS ${ }^{1}$ \\ ${ }^{1}$ Center for MicroElectroMechanical Systems (CMEMS), University of Minho, \\ 4800-058, Guimarães, Portugal \\ ${ }^{2}$ Artificial Intelligence and Computer Science Laboratory (LIACC), \\ Porto, Portugal
}

\begin{abstract}
Inertial Measurements Unit (IMU) based systems are a purposeful and alternative tool to monitor human gait mainly because they are cheaper, smaller and can be used without space restrictions compared to other gait analysis methods. In the scientific community, there are well-known studies that test the accuracy and efficiency of this method compared to ground truth systems. Gait parameters such as stride length, distance, velocity, cadence, gait phases duration and detection, or joint angles are tested and validated in these studies in order to study and improve this technology. In this article, knee joint angles were calculated from IMUs' data and they were compared with DARwIn OP knee joint angles. IMUs were attached to the left leg of the robot and left knee flexion-extension (F-E) was evaluated. The RMSE values were less than $6^{\circ}$ when DARwIn OP was walking, and less than $5^{\circ}$ when the robot kept the left leg stretched and performed an angle of $-30^{\circ}$.
\end{abstract}

Keywords: Sensor Fusion; Joint Angles; Inertial Measurement Unit (IMU).

\section{Introduction}

Gait analysis is a clinical tool for measurement, description and assessment of quantities of gait parameters that characterize human locomotion, ${ }^{1,2}$ and it is widely used to diagnose walking diseases. ${ }^{3}$ To quantify gait parameters there are several techniques ${ }^{4}$ such as image processing, ${ }^{5}$ floor sensors, ${ }^{6}$ and wearable sensors. ${ }^{7}$ Inertial Measurements Unit (IMU) systems had emerged from wearable sensors, and they are lighter, smaller, cheaper, portable, wearable, and non-invasive when compared to other gait analysis systems. ${ }^{8-10}$ Despite the fact that these sensors have advantages that attract their use in a home environment, in general, they present some small 
errors compared to other more reliable systems. For example, Leardini et al. ${ }^{10}$ validated their inertial-measurement-unit based rehabilitation system by using an 8-TV-camera stereophotogrammetric system (Vicon motion systems, UK) as ground truth system, and their root mean square error (RMSE) was less than $5^{\circ}$. Takeda et al. ${ }^{3}$ presented a method for gait analysis using wearable sensors (accelerometer and gyroscope), and they tested it in healthy subjects. As ground truth system they used a camera motion capture system, and their RMSE values were on average $6.79^{\circ}$ for knee flexion-extension (F-E). Normal range of motion (ROM) at the knee is considered to be $0^{\circ}$ of extension (stretched leg) to $135^{\circ}$ of flexion. ${ }^{11}$ Feldhege et al. ${ }^{12}$ also validated their knee angle measurement sensor system with an electro-mechanical goniometer. The calculated F-E angle of the knee joint showed a RMSE lower than $5^{\circ}$. Concerning the literature in the scientific community, although there are some studies that focus on IMUs' system validation or knee angle measurement system validation, none of them use a DARwIn OP robot as ground truth system for knee angle measurement. Besides, this article presents an IMUs' system for humanoid gait analysis. The remainder of this paper is organized as follows: in Section 2 the methods used in this experiment such as IMUs' system, DARwIn OP, calibration, validation protocol, orientation estimation, and knee joint angle measurement descriptions are presented; in Section 3 the results are demonstrated; Section 4 presents the discussion and conclusion.

\section{Methods and Materials}

\subsection{Magnetic/Inertial-based Measurement System}

A Magnetic/Inertial-based Measurement System (IMUs' System) has three main elements: a personal computer (PC), a base station, and sensory modules. Each sensory module (Fig. 1.a) is equipped with a CC2530EM (Evaluation Module) from Texas Instruments (IEEE Std 802.15.4, 2006), a MPU$6000^{13}$ from InvenSense which contains a three-axis MEMS accelerometer and gyroscope, a temperature sensor, a 3.6 Volts battery, and an antenna. A Honeywell three-axis Digital Compass IC HMC5883L ${ }^{14}$ was integrated in the MPU-6000. MPU6000, IC HMC5883L, and a battery constitute the sensors board that is connected to the CC2530EM module through two 20-pin header connectors. ${ }^{15}$ The base station is composed by a CC2530EM attached to a SmartRF05EB (Evaluation Board). This element is powered by the $\mathrm{PC}$, so its energy consumption is not a concern. Its duty is to associate new sensory modules to the network, allocate time slots on the Enhanced 
Low Power Real Time (eLPRT) superframe ${ }^{16}$ for modules to transmit, and keep the synchronization in the network. In summary, wireless communication between sensory module and the base station is made using CC2530 modules. In turn, base station sends the received data to the PC by serial port. Subsequently, the data is processed in real time computationally. The system process was simplified by the use of a Matlab Graphical User Interface (GUI) capable of informing the user in real-time about the state of the system and of performing any processing related to the data coming from the sensory modules. This interface houses all the information or process related to this system. The sampling frequency was $30 \mathrm{~Hz}$.

\subsection{Calibration Procedure}

Sensory modules were calibrated prior to trials. The method consists in three types of movements: (i) the sensory module is placed on a surface as horizontal as possible on its different faces. At each position of the sensory module the gravity constant value from the accelerometer is stored, taking into account only the sensitive axis parallel to the gravitational force; (ii) gyroscope offsets are obtained with the sensory module also placed on a surface as horizontal as possible on a static position; (iii) digital compass axes (parallel and anti-parallel) are aligned with the north of the magnetic field and maximum and minimum values are obtained. Finally, these values are compared to the sensors data from monitoring process, ${ }^{15}$ for normalization, producing outputs in the range of -1 to 1 .

\subsection{Reference Measurement System - DARwIn}

DARwIn OP (Fig. 1.a) was developed by RoMeLa ${ }^{17}$ at Virginia Tech together with Robotics Co, ${ }^{18}$ and it is a humanoid-robot platform with sophisticated sensors, advance computational power, and dynamic motion ability that enable research, education, and outreach activities. ${ }^{19}$ Users are encouraged to modify not only the hardware but also the software. On the one hand, the mechanical structure of DARwIn OP is divided into several sub-assemblies, namely: head; chest; arms; pelvis; and legs. It has 20 actuator modules with durable metallic gears, embedded sensors (3-axis gyroscope and accelerometer, and a webcam), a hardware platform to control the robot, and a battery. ${ }^{18}$ On the other hand, the robot is compatible with various programming languages, including $\mathrm{C}++$, LabView or Matlab, which allows for better interaction. It is also considered a miniature humanoid robot since its height is $454.5 \mathrm{~mm},{ }^{18}$ and has 6 degrees of freedom 
(DOFs) on each leg, 3 DOFs on each arm, and 2 DOFs on the neck. The robot also allows controlling the angle of the joints, as well as knowing the real value of the angles along the gait or any other process. ${ }^{19}$ The real values of the joints angles recorded by the robot have a low offset with respect to the theoretical value programmed in the robot. The registration of the knee joint angles in each trial will serve as ground truth to validate the IMUs' system. The data were recorded at $62.5 \mathrm{~Hz}$.

\subsection{Validation Protocol}

After the calibration process, two sensory modules were attached to the DARwIn OP left shank and thigh to acquire data (Fig. 1). Initially, as first trial, the robot started a walking process after being programmed to do so. Each trial was repeated five times for ninety seconds at one gait cycle per 2.5 seconds. Later, the robot was programmed to keep the left leg stretched on a static position (knee joint angle $=0^{\circ}$ ) for thirty-five seconds. The previous procedure was performed again at an angle of $-30^{\circ}$ as depicted in Fig. 1.b. These tests were repeated five times each. Data from the two systems were acquired simultaneously by the same PC and both are synchronized.

\subsection{Sensory Modules Orientation Estimation}

Collected data were used to estimate roll, pitch, and yaw orientation for each attached sensory module by using a complementary filter that works with normalized values from calibration procedure. ${ }^{15}$ The orientation representation can be done by Euler Angles. At this point a precise calibration is crucial, since it has a lot of influence in obtaining these estimates. Due to ferromagnetic influence from DARwIn OP structures, magnetometer was not considered in the orientation estimation. Thus, this estimation can suffer from the occurrence of drift, since the presence of this sensor would serve to correct the gyroscope measures. ${ }^{20}$

\subsection{Knee Joint Angle Measurement}

The next step is estimate the angle of the DARwIn OP left knee joint. Continuing the previous step, it is essential to use roll, pitch, and yaw (radian) from each module to achieve the angle between two planes, which are XY planes in this particular case (sensory modules axes are represented in Fig. 1.a). In order to do so, the normal vectors (Tupper - robot thigh, and Tlower - robot shank) to each plane are rotated as follows by the following rotation matrix: ${ }^{21}$ 


$$
R=R_{x}(\text { roll }) R_{y}(\text { pitch }) R_{z}(\text { yaw })
$$

Thus, from each initial normal vector $\left(\left[\begin{array}{ll}\mathrm{x} \text { y } z\end{array}\right]=\left[\begin{array}{lll}0 & 0 & 1\end{array}\right]\right)$ is obtained a new and rotated vector based on each roll, pitch, and yaw angles (in radians) estimated in the previous step. The initial vectors are rotated by the order of presentation of the rotation matrices in Eq. (1). Later, the calculation of the knee angles is performed by using these two rotated vectors

$$
\gamma=\arcsin \left(\frac{\overrightarrow{\text { Tupper }} \cdot \overrightarrow{\text { Tlower }}}{\|\overrightarrow{\text { Tupper }}\|\|\overline{\text { Tlower }}\|}\right)
$$

Where $\gamma$ is the knee angle in radians. This value is then converted to degrees, and changed to the DARwIn OP reference (e.g., to $\alpha$ in Fig. 1.b). Finally, it is only necessary to perform a resampling process so data from the robot encoders and from this estimation can be compared by calculating the RMSE. Although knee F-E is the only movement evaluated, this method can calculate three-dimensional joint angles.

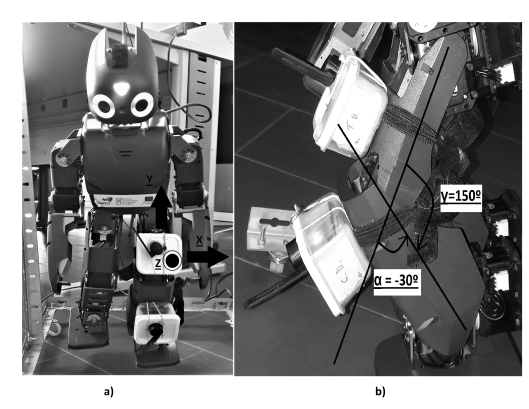

Fig. 1. (a) DARwIn robot with two sensory modules attached to left thigh and shank (for both modules, the positive $\mathrm{Z}$ axis is perpendicular to the housing cover, $\mathrm{Y}$ axis: up, $\mathrm{X}$ axis: to the left of the robot). b) DARwIn robot performs an angle of $-30^{\circ}$ (sagittal plane). Knee angles from the robot $-\alpha$, and the knee angles from the implemented model $-\gamma$.

\section{Results}

Concerning the first set of trials when the robot was walking, typical knee joint angles obtained with the encoder and the inertial-based system are displayed in Fig. 2. In this situation the RMSE value was $5.68^{\circ} \pm 0.34^{\circ}$. 
Typical results obtained for knee joint angles with both systems when the robot kept the left leg stretched are depicted in Fig. 3. The RMSE value was $4.29^{\circ} \pm 0.09^{\circ}$. When the robot performed a knee angle of $-30^{\circ}$, the RMSE value was similar to the previous one with $4.30^{\circ} \pm 0.16^{\circ}$. Typical results from both systems in this last situation are represented in Fig. 4.

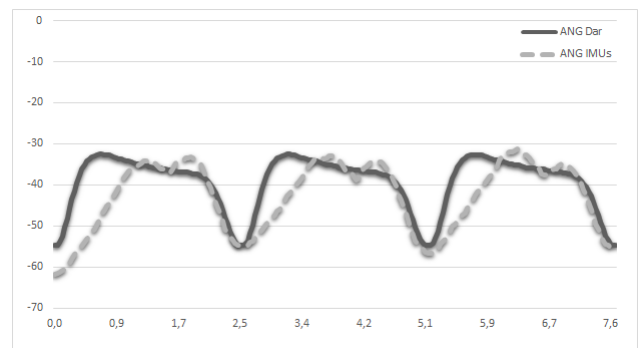

Fig. 2. Typical knee angles $\left(^{\circ}\right)$ during the trial where the DARwIn OP was walking (ANG Dar $\rightarrow$ robot real angles measured through encoders; ANG IMUs $\rightarrow$ calculated knee angles from sensory modules data; $\mathrm{x}$-axis: time (s); y-axis: angles).

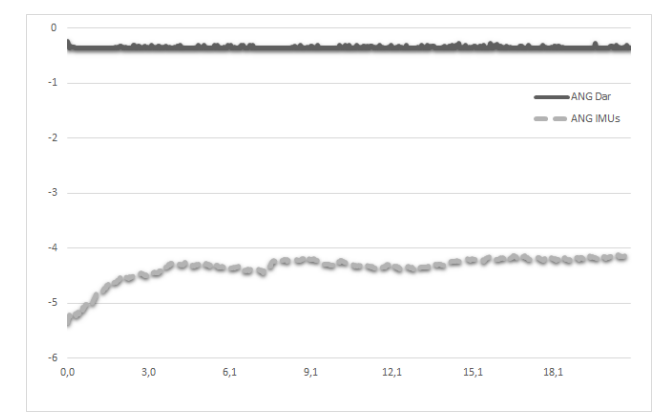

Fig. 3. Typical knee angles $\left(^{\circ}\right)$ during the trial where the DARwIn OP kept the leg stretched (ANG Dar $\rightarrow$ robot real angles measured through encoders; ANG IMUs $\rightarrow$ calculated knee angles from IMUs' data; x-axis: time (s); y-axis: angles).

\section{Discussion and conclusions}

Starting with the first trial, the RMSE value was $5.68^{\circ} \pm 0.34^{\circ}$, which is in accordance with the literature, despite being the highest value in the results. Analysing the graph of Fig. 2, it is possible to observe a slight 


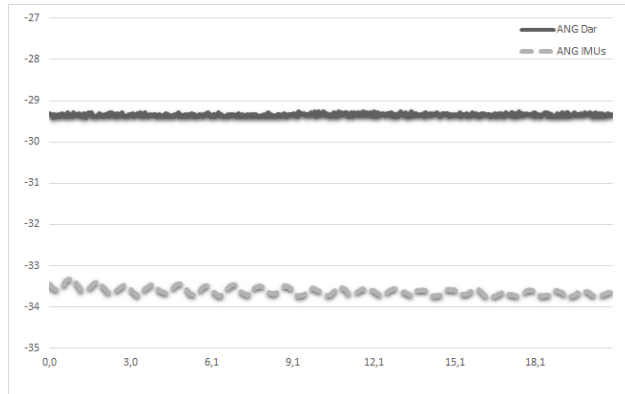

Fig. 4. Typical knee angles $\left(^{\circ}\right)$ during the trial where the DARwIn OP performed an angle of $-30^{\circ}$ (ANG Dar $\rightarrow$ robot real angles measured through encoders; ANG IMUs $\rightarrow$ calculated knee angles from IMUs' data; x-axis: time (s); y-axis: angles).

delay in the ascent. This is essentially due to a rapid transition of the segments and the model cannot follow so quickly. However, it has a similar waveform. In the other two situations, the result was lower than $5^{\circ}$, and the results are very similar to each other. Note that the real value of the angle of the robot knee joint is not exactly $0^{\circ}$ and $30^{\circ}$ in Figs. 3 and 4 , respectively. There is an offset between the ideal value and the actual value measured by DARwIn OP. In summary, the results for knee F-E angles are in accordance with the literature. This error range is within the values found in the gait analysis literature when using IMUs. In conclusion, our knee angle measurement system based on IMUs is able to be used in gait analysis, however in future work the results can be improved with other calibration methods or a post-processing procedure. In the future, Adduction/abduction and internal/external rotation angles should also be present in the validation process.

\section{Acknowledgments}

This work is supported by the FCT Fundação para a Ciência e Tecnologia - with the scholarship reference SFRH/BD/102659/2014, the reference project UID/EEA/04436/2013, by FEDER funds through the COMPETE 2020 - Programa Operacional Competitividade e Internacionalização (POCI) - with the reference project POCI-01-0145-FEDER-006941; and the LIACC Project PEst/UID/CEC/00027/2015.

\section{References}

1. W. Tao, T. Liu, R. Zheng and H. Feng, Sensors 12, 2255 (2012). 
2. S. L. Patterson, M. M. Rodgers, R. F. Macko and L. W. Forrester, The Journal of Rehabilitation Research and Development 45, 221 (2008).

3. R. Takeda, S. Tadano, A. Natorigawa, M. Todoh and S. Yoshinari, IFMBE Proceedings 25, 111 (2009).

4. A. Muro-de-la Herran, B. Garcia-Zapirain and A. Mendez-Zorrilla, Sensors (Switzerland) 14, 3362 (2014).

5. J. Chen and J. Liu, Scientific World Journal 2014 (2014).

6. A. L. McDonough, M. Batavia, F. C. Chen, S. Kwon and J. Ziai, Archives of Physical Medicine and Rehabilitation 82, 419 (2001).

7. A. Sant' Anna, N. Wickstrum, H. Eklund, R. Zugner and R. Tranberg, Assessment of Gait Symmetry and Gait Normality Using Inertial Sensors: InLab and In-Situ Evaluation, in Communications in Computer and Information Science, eds. J. Gabriel, J. Schier, S. Van Huffel, E. Conchon, C. Correia, A. Fred and H. Gamboa (Springer Berlin Heidelberg, Berlin, Heidelberg, 2013), Berlin, Heidelberg, pp. 239-254.

8. L. Ambrozic, M. Gorsic, S. Slajpah, R. Kamnik and M. Munih, Wearable sensory system for robotic prosthesis (1) (International Journal of Mechanics and Control, 2014).

9. T. Liu, Y. Inoue and K. Shibata, Measurement: Journal of the International Measurement Confederation 42, 978 (2009).

10. A. Leardini, G. Lullini, S. Giannini, L. Berti, M. Ortolani and P. Caravaggi, Journal of NeuroEngineering and Rehabilitation 11, 136 (2014).

11. N. P. Fey, A. M. Simon, A. J. Young and L. J. Hargrove, IEEE Journal of Translational Engineering in Health and Medicine 2 (2014).

12. F. Feldhege, A. Mau-Moeller, T. Lindner, A. Hein, A. Markschies, U. K. laus Zettl and R. Bader, Sensors (Switzerland) 15, 10734 (2015).

13. InvenSense, MPU-6000 and MPU-6050 Product Specification, (2013).

14. Honeywell, 3-Axis Digital Compass IC, (2011).

15. P. Macedo, J. A. Afonso, L. A. Rocha and R. Simoes, A Telerehabilitation System Based on Wireless Motion Capture Sensors (PhyCS - Proceedings of the International Conference on Physiological Computing Systems, 2014).

16. J. A. Afonso, H. D. Silva, P. Macedo and L. A. Rocha, Sensors 11, 3852 (2011).

17. RoMeLa (Robotics \& Mechanisms Lab), DARwIn OP: Open Platform Humanoid Robot for Research and Education.

18. Trossen Robotics, Darwin-OP Humanoid Research Robot - Deluxe Edition.

19. T. Pham, S. Rouleau, C. Knabe, M. Stanford, N. Woldesemayat, M. Umansky, J. S. Austin, A. Kim, A. McCormack, D. Branch and V. Orekhov, DARwIn OP Fabrication Manual, (2011).

20. A. Laudanski, B. Brouwer and Q. Li, Journal of Healthcare Engineering 4, 555 (2013).

21. F. Dunn, I. Parberry and A. Fallis, 3D Math Primer for Graphics and Game Development, 2nd Edition, 2nd edn. (A K Peters/CRC Press 2011, 2011). 